\title{
Manufacturing and testing flexible microfluidic devices with optical and electrical detection mechanisms
}

\author{
Marius G. Ivan*, Frédéric Vivet, and Erwin R. Meinders \\ Holst Centre/ TNO, High Tech Campus 31, 5656 AE, Eindhoven, Netherlands
}

\begin{abstract}
Flexible microfluidic devices made of poly(dimethylsiloxane) (PDMS) were manufactured by soft lithography, and tested in detection of ionic species using optical absorption spectroscopy and electrical measurements. PDMS was chosen due to its flexibility and ease of surface modification by exposure to plasma and UV treatment, its transparency in UV-Vis regions of the light spectrum, and biocompatibility. The dual-detection mechanism allows the user more freedom in choosing the detection tool, and a functional device was successfully tested. Optical lithography was employed for manufacturing templates, which were subsequently used for imprinting liquid PDMS by thermal curing. Gold electrodes having various widths and distances among them were patterned with optical lithography on the top part which sealed the microchannels, and the devices were employed for detection of ionic species in aqueous salt solutions as well as micro-electrolysis cells. Due to the transparency of PDMS in UV-Vis the microfluidics were also used as photoreactors, and the in-situ formed charged species were monitored by applying a voltage between electrodes. Upon addition of a colorimetric $\mathrm{pH}$ sensor, acid was detected with absorption spectroscopy.
\end{abstract}

Keywords: microfluidics, flexible, disposable cartridges, soft lithography, photolithography, dual detection, UV-Vis spectroscopy, conductivity, sensors, electrolysis cell, micro-photoreactors

\section{INTRODUCTION}

Microfluidic devices have spun a wide range of applications in recent years, sparked by advances in microelectronics, fabrication techniques, and novel materials.[1, 2] Applications range from analytical chemistry to separation of chemical species, microreactors[3], detection of biologically-relevant molecules[2, 4-6], cell counting, routine blood analysis[7], food control, and combinatorial chemistry with applications in pharmaceutical industry, etc. While in the 1990's the first microfluidic devices were manufactured in glass, quartz or silicon, there has been a shift towards softer lithography methods and polymeric materials in the past decade.[1, 8-11] Advantages offered by polymeric materials are numerous, and they stem from ease of manufacturing, i.e. injection molding, casting and hot embossing[12], to low cost associated with manufacturing and exploitation, biocompatibility and higher flexibility, which allows easier integration of electrical and mechanical components. [8, 12-14] Polymers also allow easy modification of surfaces by grafting functional sensors or molecules required for various applications, such as surface energy modification. Poly(dimethylsiloxane) is the most common material employed for manufacturing polymeric microfluidic devices, and was previously reported by others.[1] $[8,9,12]$

Our goal on the long run is to manufacture microfluidics with a low-manufacturing cost, in a roll-to-roll fashion. Thus the materials, components, and parts would have to be flexible, bendable, and solution processable.

In the present paper we report on manufacturing and testing of flexible poly(dimethylsiloxane) (PDMS) microfluidic cartridges and attempts to manufacture poly(ethylene terephthapate) (PET) microfluidic devices targeted at analytical detection and quantification of various chemically or biologically-relevant species. PDMS is a hydrophobic material, with water contact angles measured at $>110^{\circ}$, thus not ideal for aqueous solutions, so we attempted to use PET which is a hydrophilic material. The detection mechanism may be dual - optical and electrical, making it versatile detection device. [15] Optical detection is based on absorption or transmittance spectroscopy in UV-Vis regions of the electromagnetic spectrum, and allows for quick tests as well as time-based measurements. This type of detection may be used when analytes are also chromophores with known absorption bands, or by delivering solutions with sensors capable of

*marius.ivan@tno.nl, ; phone+31 40277 4348; www.holstcentre.com,www.tno.nl

Photonics North 2010, edited by Henry P. Schriemer, Rafael N. Kleiman, Proc. of SPIE Vol. 7750 , $775002 \cdot$ ? 2010 SPIE $\cdot$ CCC code: 0277-786X/10/\$18 - doi: 10.1117/12.870318 
changing their absorption or fluorescence spectra upon interaction with the analytes. Electrical detection adds up extra functionality and offers extra choices for the user in the event that ionic solutions are analyzed. Because of the Au electrodes that come in contact with the liquid, such a device may be used as a micro-electrolysis cell, with the possibility of locally forming chemical reagents. We demonstrate the use of a PDMS device as a micro-photoreactor, and monitor the formation of ionic species upon the photolysis of a photoacid generator.

\section{EXPERIMENTAL}

A process was developed to manufacture plastic microfluidic cartridges based on soft lithography. Templates were used to structure the microfluidic channels via thermal curing of the casted polymer solution or by hot embossing. The metal electrodes were subsequently patterned on another plastic foil via optical lithography, and then the microfluidic device was obtained by bonding the two separate parts. Proper functioning of the device was tested by measuring the absorption spectra of Coumarin 6, a pH sensor, with a UV-Vis spectrophotometer, and by monitoring the current vs. voltage for distilled water and solutions containing $\mathrm{NaCl}$.

\subsection{Template development}

Two types of templates were developed and tested: Si- and resist-based, with their use dependent on the type of materials from which the devices were manufactured, and the imprinting method. Si templates were employed for hot embossing, while resist-based templates for soft lithography.

Si templates. The templates were made from 4" and 6" Si wafers via photolithography. The 4" Si wafers were employed during the hot embossing step with a Specac press. Masks for i-line optical lithography bearing the pattern of the microfluidic channels were obtained first, and then their pattern was projected with the help of a Karl Suss MA8 mask aligner on a thin film of resist (HPR $504 \sim 1.0 \mu \mathrm{m}$ ), deposited on a 4" Si wafer. The incident dose was $80 \mathrm{~mJ} / \mathrm{cm}^{2}$. The wafers were then baked on a hot plate and developed for $60 \mathrm{~s}$ in a solution of developer PLSI : water $=1 / 1$ volume ratio. The wafers were cleaned with demineralized water, air-dried and the remaining organic impurities in the developed areas were removed during an $\mathrm{O}_{2}$ plasma treatment. Si dry etch was performed with an STS Multiplex until a depth of $45 \mu \mathrm{m}$ was reached, see Fig. 3 and 4. The remaining resist was removed in acetone and during a descum plasma treatment.

Resist-based templates. While Si templates are robust and suitable for imprint experiments where high temperatures and pressures are employed, for faster and lower cost manufacturing of PDMS microfluidic devices we decided to use a different approach. Previous reports in literature mention SU8 as resist of choice for obtaining microfluidic templates. [8, 16] Because of the polarity of our mask, a layer of AZ 9260 positive photoresist from AZ Chemicals was spin coated on top of 6" Si wafers for $60 \mathrm{~s}$ at $2100 \mathrm{rpm}$ (rotations per minute). In order to reach a thickness of $\sim 40.0 \mu \mathrm{m}$, the deposition was done in four steps, each time employing the same deposition parameters, followed by a post-application bake at 110 oC for 6 minutes. The wafers coated with resist were exposed to i-line radiation in a Karl Suss MA8 mask aligner to an energy dose of $2700 \mathrm{~mJ} / \mathrm{cm}^{2}$. Development was done with AZ $400 \mathrm{~K}$ for 15 minutes. After resist development, the sample was cleaned with demineralized water, then dried, and let for $24 \mathrm{~h}$ inside a desicator containing a volatile fluorinated compound, perfluorodecyltrichlorosilane (PFDTS). Due to its high content of fluorine, this compound acts as an antisticking layer, facilitating easy removal of the PDMS from the template. On a 6" wafer we crammed twenty devices with dimensions of $2.0 \times 3.0 \mathrm{~cm}^{2}$ with the same layout as the one presented in figure 1 .

\subsection{Manufacturing microfluidic devices}

Fabrication of microchannels. The substrates with microfluidic structures were made from poly(dimethylsiloxane) (PDMS), commercially known as Sylgard 184 silicone elastomer from Dow Corning, consisting of base and curing agent which were mixed in a 10:1 weight ratio. The freshly prepared solution was casted on top of the Si- or resist-based templates, allowed for air bubbles removal at room temperature for 10 minutes, and then baked at $100{ }^{\circ} \mathrm{C}$ for 1 hour, ensuring cross-linking of PDMS. After baking, the sample was cooled down to room temperature on a metallic plate and then peeled-off from the template.

Fabrication of the part containing Au electrodes. The part containing the Au electrodes, layer 2 in Fig. 1, was obtained by employing the so-called foil-on-carrier method.[17] First a $20 \mu \mathrm{m}$ thick layer of thermally curable adhesive was deposited by spin-coating on top of a $6 " \mathrm{Si}$ wafer, and then pre-cured at $100{ }^{\circ} \mathrm{C}$ for $30 \mathrm{~s}$. A $25 \mu \mathrm{m}$ thick poly(ethylenenaphthalate) (PEN) foil, Q65FA Teonex from DuPont Teijin Film, was attached to the top of the adhesive 
layer by applying pressure with a rotating cylinder at a controlled speed. The stack was then baked at $100 \mathrm{oC}$ on a hot plate for 45 minutes, ensuring the cross-linking of the adhesive. A solution of Sylgard 184 polymer and curing agent in a weight ratio of 10:1 was spin-coated on top of the PEN foil, affording a $\sim 20 \mu \mathrm{m}$ thick film, followed by thermal curing of the PDMS layer. On top of this stack a $5.0 \mathrm{~nm}$ Ti layer was deposited, followed by deposition of $50 \mathrm{~nm}$ of Au. Two methods were employed for Au deposition: sputtering and electron-beam physical vapor deposition (EBPVD).

Patterning electrodes. Au electrodes were patterned with optical lithography on top of a thin PDMS layer. A thin layer of $\mathrm{Au}, 50 \mathrm{~nm}$, was deposited by evaporation with a BAK 500 on a PDMS layer spin-coated on top of a PEN foil, laminated on a 6" Si wafer. The Si wafer offers dimensional stability to the plastic foil, and allows processing with the tools available in the clean room. Prior to Au evaporation, a $5 \mathrm{~nm}$ Ti layer was deposited, with the aim of enhancing Au adhesion to the substrate. Then HPR photoresist was spin coated on top of the Au to afford a $1.0 \mu \mathrm{m}$ thick layer, baked at $90{ }^{\circ} \mathrm{C}$ for 2 minutes, followed by exposure through a mask with a Karl Süss MA8 mask aligner to $80.0 \mathrm{~mJ} / \mathrm{cm}^{2}$ i-line radiation. After exposure the wafers were developed with PLSI developer, and then subjected to wet etching of Au and Ti layers with $\mathrm{KI} / \mathrm{I}_{2}$ and a solution of $\mathrm{HF} / \mathrm{HNO}_{3} / \mathrm{H}_{2} \mathrm{O}$ respectively. Resist stripping with acetone completed the fabrication process of the Au electrodes.

PDMS bonding. The two parts of the microfluidic device- the bottom part containing the microchannels and the top part containing the Au electrodes- were bonded employing a procedure previously reported by others.[1] Initial attempts to employ poly(ethylenenaphthalate) (PEN) as top cover were not successful, as the PDMS devices delaminated when liquid was delivered into the microchannels. We chose PEN due to our previous experience on patterning metal layers on PEN substrates with optical lithography, and ease of patterning.[17] Even though PEN films were subjected to an $\mathrm{O}_{2}$ plasma, the bonding to the PDMS device was not strong enough. The best adhesion to the PDMS layer would be when another plasma-treated PDMS layer is employed.[8] We decided to pattern Au electrodes on top of a PDMS membrane, and then bond the two parts after $\mathrm{O}_{2}$ plasma treatment (see experimental part for Au electrodes patterning). The PEN foil was employed as carrier of the PDMS layer on top of which the Au electrodes were patterned, and offers the advantage of an easy manipulation of PDMS films and avoids contamination. The two parts of the device -microchannels containing bottom and electrodes containing top part-were subjected to an $\mathrm{O}_{2}$ plasma treatment, which leads to the formation of silanol $(\mathrm{Si}-\mathrm{OH})$ groups on the surface.[16, 18] Immediately after plasma treatment, the two surfaces were brought in tight contact, with formation of an irreversible sealing due to chemical bonds Si-O-Si. Considering that PEN is opaque below $380 \mathrm{~nm}$, for certain optical sensors it may be replaced with poly(ethyleneterephthalate) (PET) which is opaque below $360 \mathrm{~nm}$, thus expanding the monitoring interval.

\subsection{Materials and methods}

Coumarin 6, a pH sensitive fluorescent dye was purchased from Sigma-Aldrich and used as received. The solution employed for UV-Vis absorption spectroscopy tests was $1.17 \times 10^{-3} \mathrm{M}$ Coumarin 6 in acetonitrile, and the baseline was the empty microfluidic device. UV-Vis measurements were performed with a U-2010 spectrophotometer from Hitachi. $\mathrm{NaCl}$ from Aldrich was dissolved in distilled water. The photoacid generator (4-methoxyphenyl)-diphenylsulfonium triflate was purchased from Aldrich and used as received. A Hewlett Packard probe station was employed for the use of the device as a micro-electrolysis cell and measurements of the current $v s$. voltage for ionic solutions by applying a voltage on the two Au pads. Profilometry measurements were performed with a Dektak tool from Veeco, and scanning electron microscopy images were recorded with XL40 from FEI/Philips. UV exposures were performed inside a photoreactor with top-down irradiation.

\section{RESULTS AND DISCUSSION}

As a proof of principle, we opted for a simple design of the microfluidic device, as shown in Fig. 1, however we could potentially obtain much more complex microfluidic devices. Two inlets, $0.5 \mathrm{~mm}$ in diameter, were continued through microchannels, $80.0 \mu \mathrm{m}$ in width. The first two microchannels were joined, and the resulting microchannel had a width of $100.0 \mu \mathrm{m}$, with seven loops which allow better mixing of the liquids delivered. A square monitoring window, $2.0 \mathrm{~mm}$ x $2.0 \mathrm{~mm}$, was created for UV-Vis spectroscopy measurements, which ended up with an outlet for liquid removal. On the top cover of the microfluidic device we deposited two electrodes with a gap varying between $20-100 \mu \mathrm{m}$, and the electrode width $20.0 \mu \mathrm{m}$. For easy contact with the probe needles, we designed two pads at the end of each electrode, see Fig. 1. The top side containing the electrodes is slightly wider than the side containing the microchannels, making thus the pads easily accessible for the electrical measurements with the probe needles. This ensured that the devices are not 
punctured when electrical measurements were performed. The dimensions of layer 1 are $2.0 \times 3.0 \mathrm{~cm}^{2}$, while layer 2 is $3.0 \times 4.0 \mathrm{~cm}^{2}$. Images of an actual device are displayed in Fig. 6 .

Two masks corresponding to the two overlapped layers in Fig.1 (left) were obtained on glass substrates: first mask with the layout of the microfluidic channels, inlets, monitoring window and outlet, and a second mask with the electrodes layout. The mask with layer 1 was employed for manufacturing the imprint template. The second mask was employed for patterning Au electrodes on top of a thin PDMS layer with optical lithography.
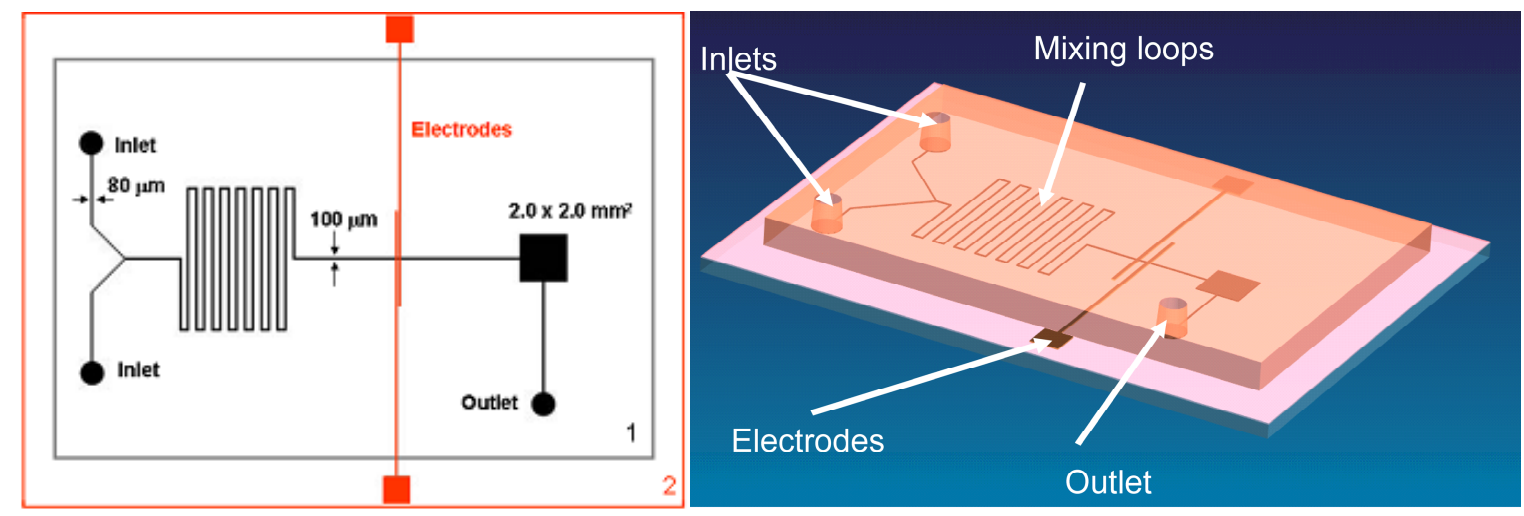

Fig. 1. Schematic of the two layers comprising the microfluidic device(left side): two inlets ( $0.5 \mathrm{~mm}$ diameter), two microchannels from inlets $(80 \mu \mathrm{m}$ wide) which join to form one common channel (100 $\mu \mathrm{m}$ wide), seven loops, monitoring window for UV-Vis spectroscopy $\left(2.0 \times 2.0 \mathrm{~mm}^{2}\right)$. The dimensions of the microfluidics cartridge are $2.0 \mathrm{x}$ $3.0 \mathrm{~cm}^{2}$. The $\mathrm{Au}$ electrodes $(20 \mu \mathrm{m}$ wide, with gaps of 20,50 , and $100 \mu \mathrm{m})$ are patterned on a second PDMS membrane slightly wider than the base to which it is subsequently bonded, allowing the pads to be easily contacted by the tip of the probe. A $3 \mathrm{D}$ representation of the device is displayed on the right side of the figure.

\subsection{Manufacturing and testing $\mathrm{Si}$ and resist templates}

Si templates manufactured according to the procedure described in the Experimental Section were selected for experiments during the first part of the project, where poly(ethyleneterephthalate) (PET) films were imprinted by hot embossing. The $100 \mu \mathrm{m}$ thick PET films (Agfa PET V109) were imprinted with 4" Si templates at $150{ }^{\circ} \mathrm{C}$ and 12, 20 , and 24 bars respectively for 20 and 40 minutes. The imprint temperature was by $\sim 70{ }^{\circ} \mathrm{C}$ higher than the $\mathrm{Tg}$ of the polymer, and the template was released from the substrate at $65^{\circ} \mathrm{C}$, below the $\mathrm{Tg}$ of $\operatorname{PET}\left(\sim 80^{\circ} \mathrm{C}\right)$.

The SEM images of the Si template, Fig. 2 and the recorded profile, Fig. 3, show clearly defined microchannels with a depth of $\sim 45 \mu \mathrm{m}$ and a full width at half maximum of $\sim 100 \mu \mathrm{m}$.
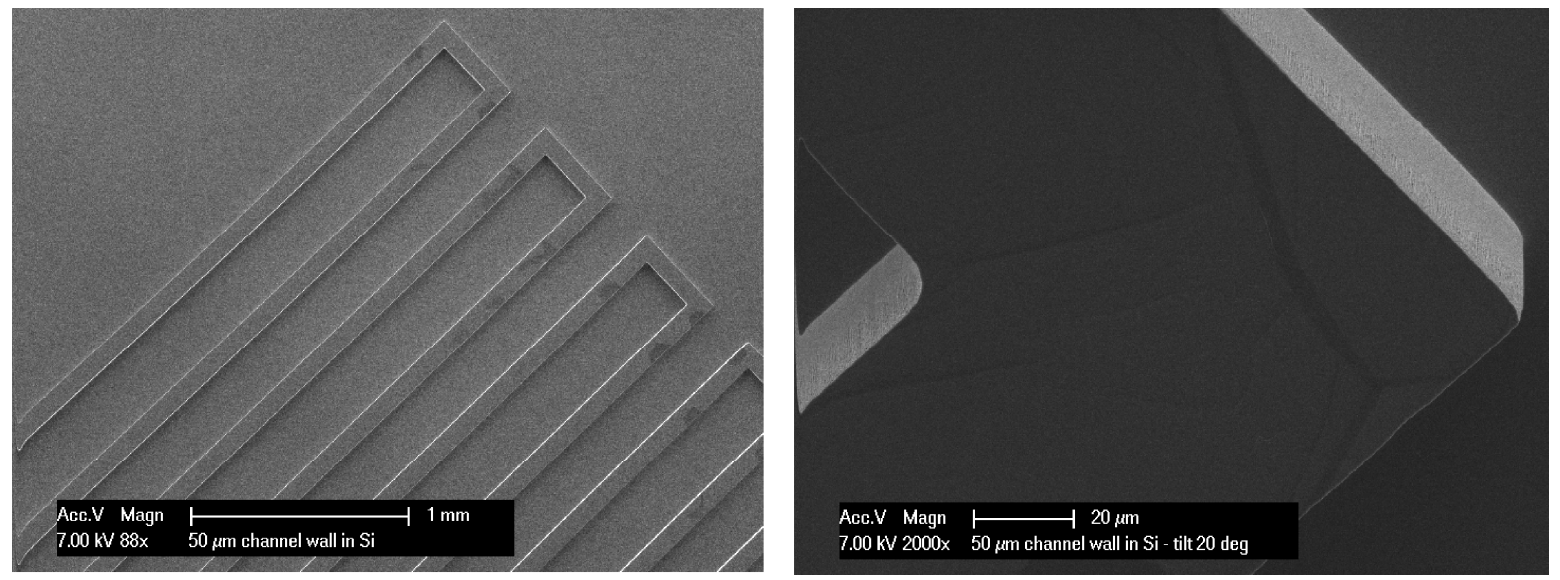

Fig. 2. SEM images of Si template after etching and stripping the remaining HPR 504 resist. The scale bars are $1.0 \mathrm{~mm}$ (left) and $20.0 \mu \mathrm{m}$ (right) 
The hills in the SEM images and the profilometry graph correspond to the microchannels in the imprinted polymeric materials.

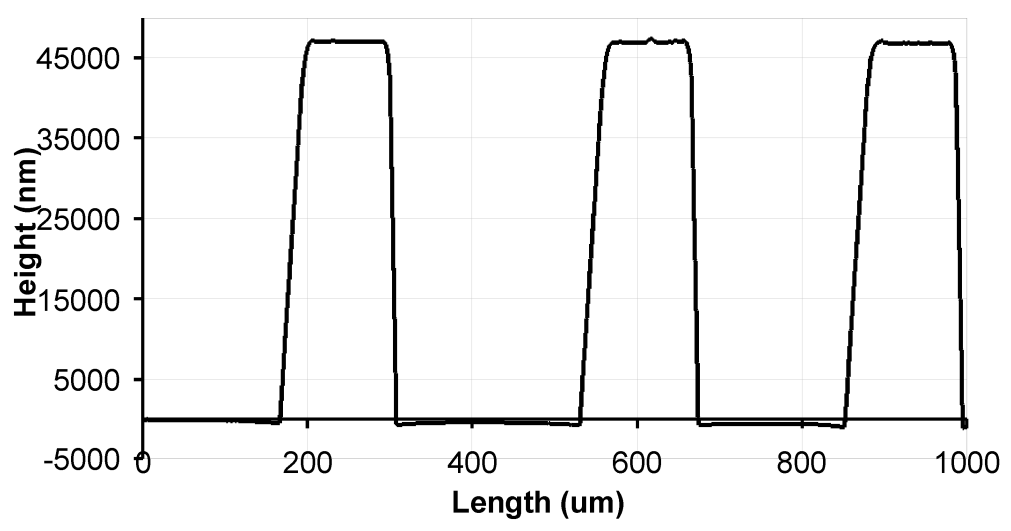

Fig. 3. Surface profilometry of a Si template, with a height of $\sim 45 \mu \mathrm{m}$, and a FWHM of $\sim 100 \mu \mathrm{m}$. The units are $\mathrm{nm}$ and $\mu \mathrm{m}$ for the $\mathrm{y}$ and $\mathrm{x}$ axis respectively.

The profile of the imprinted PET film showed incomplete filling of the template even after prolonged imprinting at high temperature and pressure. The imprint temperature was $150^{\circ} \mathrm{C}$ and the demolding temperature of $65^{\circ} \mathrm{C}$. We chose this imprint temperature because it is higher than the glass temperature of PET, and the higher the material was heated, the softer it became; however at temperatures higher than $150^{\circ} \mathrm{C}$ the foil became irreversibly modified. The demolding temperature was always kept at $65^{\circ} \mathrm{C}$, since this temperature had to be lower than the glass transition temperature in order to keep the imprinted profile during the releasing step.

The first attempt was performed at a pressure of 12 bars, and the profiles of the imprinted PET foil showed that the filling of the template features by PET was poor. For the following experiment we chose to increase the pressure to 20 bars. Even this pressure was not enough to reach a good replication of the features, and higher pressures caused the templates to break. The incomplete filling was most likely caused by the relatively large volume of displaced material which was needed to fill the large feature sizes in the template. It is obvious that at $70{ }^{\circ} \mathrm{C}$ above its $\mathrm{Tg}$, the PET foil still showed a high viscosity, which caused incomplete filling.

After $\mathrm{O}_{2}$ plasma treatment of the PET substrates, the attempts to laminate the foil with imprinted channels and the part with electrodes failed, and leakage was noticed when solutions were injected into the microchannels.

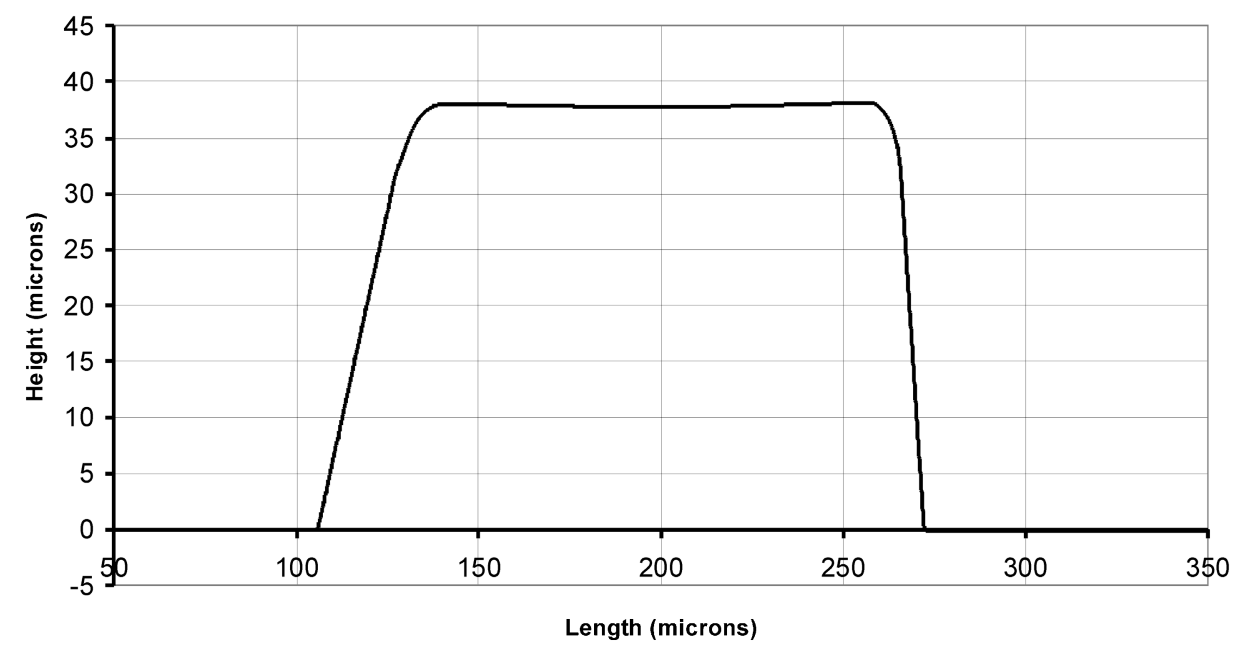

Fig. 4. Surface profilometry of an AZ 9260 resist template, with a height of $\sim 37 \mu \mathrm{m}$, and a FWHM of $\sim 140 \mu \mathrm{m}$. 
Resist-based templates were manufactured by employing a thick positive resist, AZ 9260, on top of 6" Si wafer. After exposure and development, the profile recorded shows a height of $\sim 37 \mu \mathrm{m}$ and a width of $\sim 140 \mu \mathrm{m}$, Fig. 4 .

The resist templates were replicated in poly(dimethylsiloxane) (PDMS) according to the procedure described in the experimental section. The PDMS microchannels were then studied with optical microscopy and SEM, and showed formation of microchannels with the desired depth and width, Fig. 5. PDMS has been previously employed by others in manufacturing microfluidic cartridges and requires, energy wise, less drastic conditions than hot embossing.[1, 8, 9, 12]
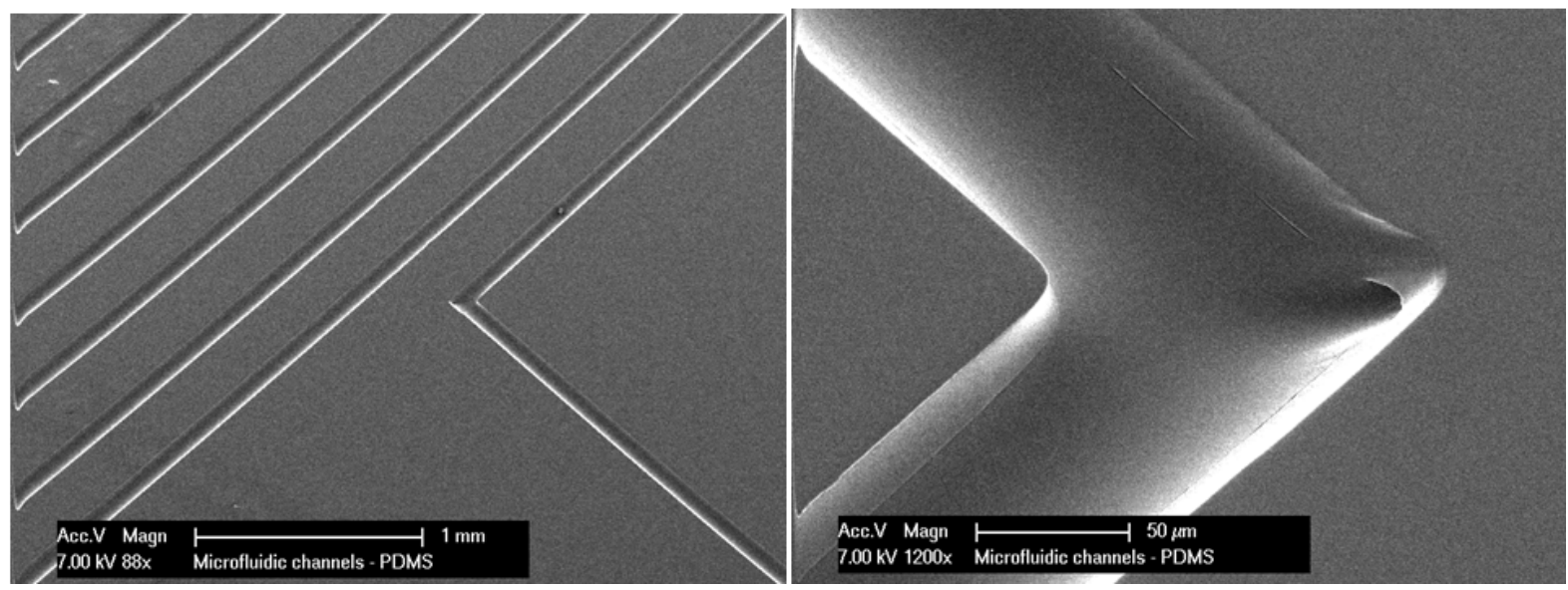

Fig. 5. SEM images of PDMS microfluidic channels obtained with resist AZ 9260 templates, with a depth of $37 \mu \mathrm{m}$; the scale bars represent $1.0 \mathrm{~mm}$ (left) and $50 \mu \mathrm{m}$ (right) respectively.

\subsection{Au electrodes}

Two deposition methods were employed for Au electrodes: sputtering and evaporation. Sputtering causes formation of wrinkles, leading to a poor quality of the Au layer, Fig. 6A. However, when vapor deposition was employed, the PDMS surface and the Au electrodes show a flat surface, without topography, Fig. 6B. A potential cause for the formation of wrinkles may be the stress induced in the PDMS layer during the deposition process. The adhesion of Au deposited by sputtering is much stronger than in the case of evaporation, thus any stress in the metal layer may be easier transferred to the underlying polymer layer.
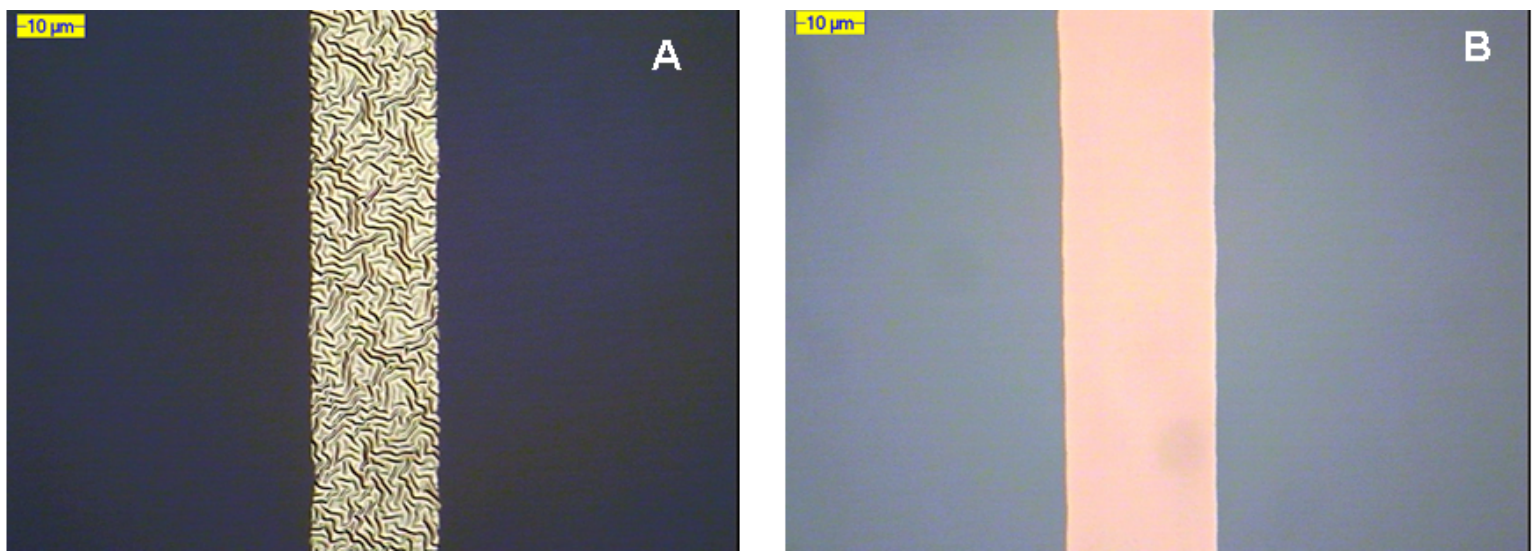

Fig. 6. Optical microscope images of $20 \mu \mathrm{m}$ Au electrodes deposited on top of PDMS by sputtering (A) and by evaporation (B), obtained with optical lithography. 


\subsection{Flexible microfluidic devices}

Obviously the resist-based templates are suitable for procedures where high pressure is not required, and solvents of the casted solution do not dissolve the template. Hardening the template by thermal and radiation treatments, combined with a fast crosslinking reaction of the casted polymer may expand the use of these templates to other polymeric solutions as well. The two layers were laminated after $\mathrm{O}_{2}$ plasma treatment, and flexible microfluidics devices were obtained, Fig. 7.
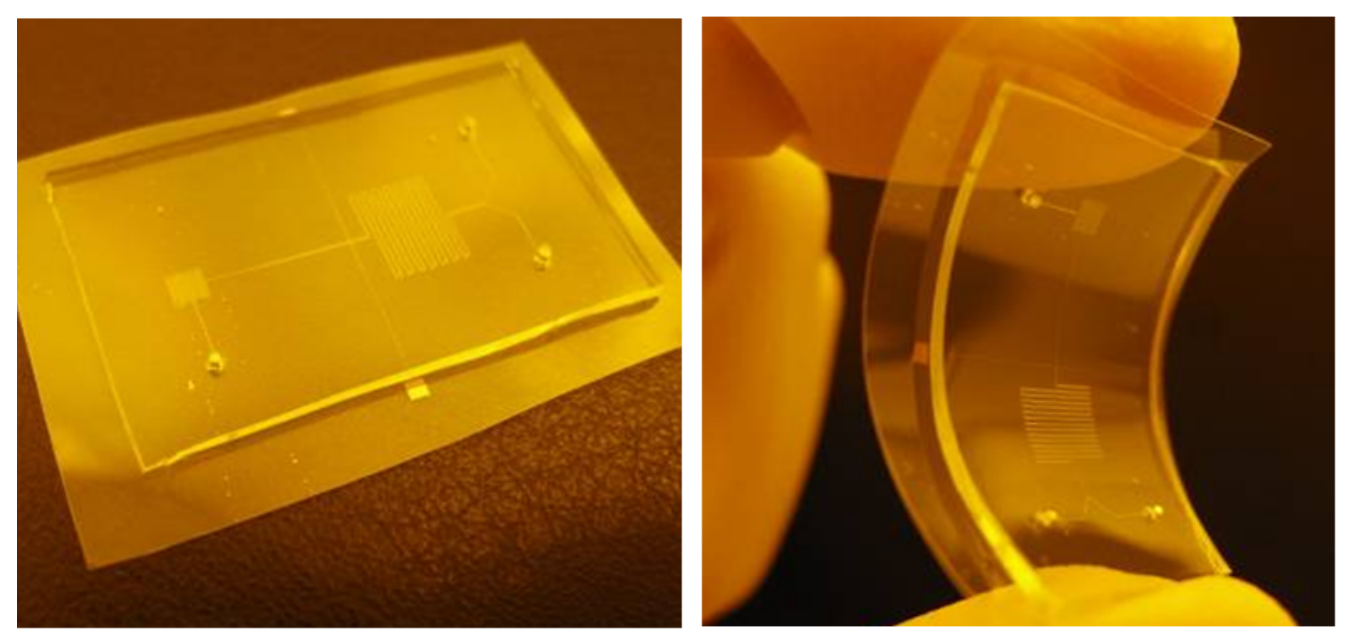

Fig. 7. Photographs of flexible microfluidic devices obtained in PDMS, with Au electrodes across the microchannels for measurements of current intensity in ionic solutions and a $2.0 \times 2.0 \mathrm{~mm}^{2}$ monitoring window for UV-Vis absorption spectroscopy.

\subsection{Testing microfluidic devices for $\mathrm{UV}$-Vis spectroscopy}

A versatile microfluidic device that offers the possibility of detecting analytes by means of monitoring optical and electrical signals may offer the user more options for quantification of various species. Devices manufactured by employing the resist-based templates were tested and were shown to function as micro-electrolysis cells and as microphotoreactors.

For UV-Vis absorption spectroscopy we selected Coumarin 6, a known $\mathrm{pH}$ sensor which changes its absorption and fluorescence maxima upon protonation of its $\mathrm{N}$ atoms, Scheme 1.[19, 20] In neutral form, Coumarin 6 absorbs with a maximum centered at $\sim 450 \mathrm{~nm}$. Upon addition of low quantities of acid, monoprotonation at the $\mathrm{N}$ atom in the heterocyclic ring occurs first, accompanied by a shift in absorption maximum to $520 \mathrm{~nm}$. Addition of more acid leads to double protonation of Coumarin 6 , with a new maximum centered at $\sim 410 \mathrm{~nm}$. Because of the large shifts in absorption maxima, Coumarin 6 was selected for testing the PDMS microfluidic devices with UV-Vis absorption spectroscopy. Another reason was its absorption in UV-Vis above $380 \mathrm{~nm}$, the wavelength below which the PEN foil which acts as support for the Au electrodes is opaque.

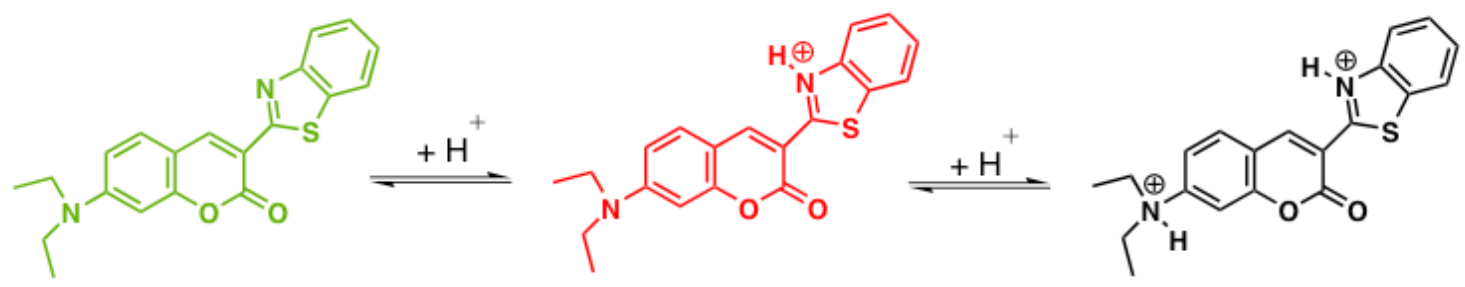

Scheme 1. Chemical reactions involving Coumarin 6: monoprotonation of the neutral form (left) occurs at the $\mathrm{N}$ atom in the heterocyclic ring (middle), and diprotonation involves the second $\mathrm{N}$ atom in the diethylamine moiety (right). 
A solution of neutral Coumarin 6 dissolved in acetonitrile, $1.72 \times 10^{-3} \mathrm{M}$, was delivered with a syringe into the microchannels of a device, then the absorption spectra was recorded, with the device positioned such that the monitoring beam crossed through the square monitoring window, figure 8 . We decided to use a fairly concentrated solution, considering that the thickness of the liquid layer in the monitoring window is $\sim 45 \mu \mathrm{m}$. We recorded an absorbance of $\sim$ 0.08 for the solution containing neutral Coumarin 6 , and the baseline was the empty microfluidic device. Acetonitrile is virtually transparent in this region of the light spectrum. Upon addition of hydrochloric acid, the two peaks corresponding to mono- and di-protonated Coumarin 6 were noticed, caused by the acid delivered in an amount enough to partially double protonate the dye, resulting in two species simultaneously present in solution.

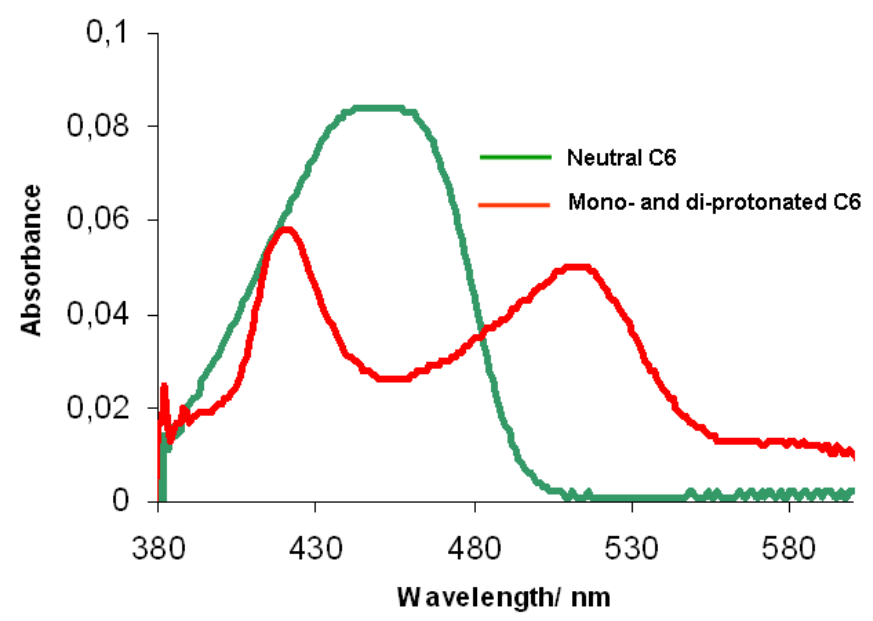

Fig. 8. Absorption spectra of neutral (green) and mono- and di-protonated (red) Coumarin 6. The absorption maxima correspond to neutral form $(450 \mathrm{~nm})$, monoprotonated $(520 \mathrm{~nm})$, and diprotonated $(418 \mathrm{~nm})$. The concentration of Coumarin 6 in acetonitrile was $1.72 \times 10^{-3} \mathrm{M}$, and the mono-and di-protonated forms were obtained upon addition of $\mathrm{HCl} 37 \%$ in water.

Quantitative measurements may be done upon calibration with known concentrations of hydrochloric acid. Besides checking the suitability for optical spectroscopy, our goal here was to test the resistance of such a device when organic solvents and acids are delivered to the microchannels. The two parts of the device did not delaminate during experiments, and adhesion tests revealed a strong bonding even one year after the devices were fabricated.

\subsection{Microfluidics as electrolysis cells}

Solutions of $\mathrm{NaCl}$ of various concentrations were delivered with a microsyringe into the microchannels, and the current intensity was measured with a Hewlett Packard probe as a function of voltage, concentration, and electrodes gap.

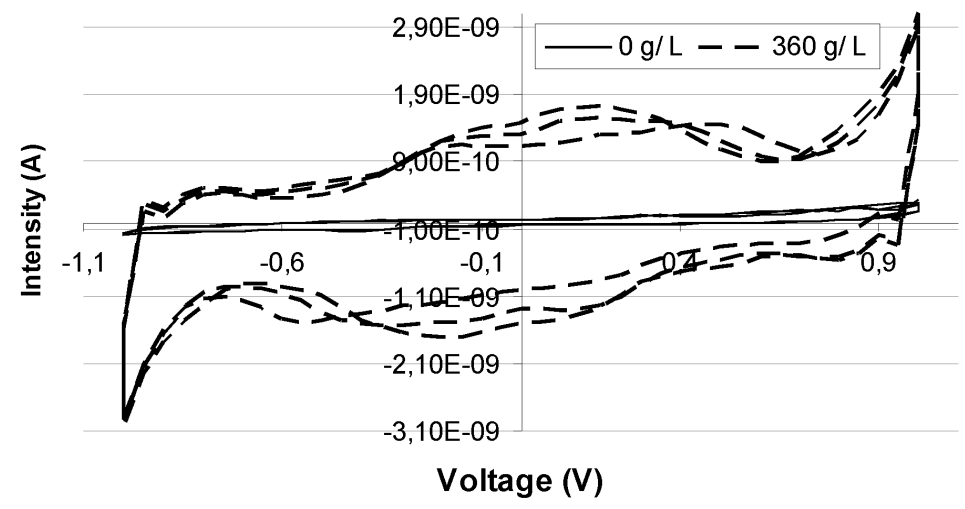

Fig. 9. Current vs. voltage for a microfluidic device with distilled water (continuous line) and $360.0 \mathrm{~g} \mathrm{NaCl} / \mathrm{L}$ (dashed line), with an electrodes gap of $100.0 \mu \mathrm{m}$. 
For the control experiment, the plot in Fig. 9 shows a very low current for the $-1.0-1.0 \mathrm{~V}$ interval when distilled water was delivered to the microdfuidics device with a $100.0 \mu \mathrm{m}$ electrodes gap. The residual conductivity may be due to ionic contaminants found in water. When a saturated solution of $\mathrm{NaCl} 360 \mathrm{~g} / \mathrm{L}$ was delivered into the channels, the readings show current an order of magnitude higher when voltage is applied. The device behaves in fact similar to an electrolysis cell, with formation of $\mathrm{Cl}_{2}$ and $\mathrm{NaOH}$ at electrodes. This may be exploited for in-situ synthesis of certain reagents based on redox reactions at electrodes.

\subsection{Microfluidics employed as micro-photoreactors}

A solution of (4-methoxyphenyl)-diphenylsulfonium triflate $1.58 \times 10-3 \mathrm{M}$ in ethanol was delivered with a microsyringe into the microchannels of a PDMS device, and then exposed to UV light inside a photoreactor for various time intervals $(0,4,6$, and 8 minutes). The same sample was successively exposed and then the current (A) vs. the applied tension (V) was recorded, figure 10. Upon absorption of photons and photolysis, the PAG yields triflic acid and other reaction products, Scheme 2. The increase in triflic acid is noticed in the increase in current as a function of the exposure time, and after 8 minutes of exposure a decrease is noticed. This could potentially be caused by the decrease in the concentration of protons which may be reduced to molecular $\mathrm{H}_{2}$ during the time that a voltage is applied. Other causes may be reactions with intermediates or products formed during the exposure.

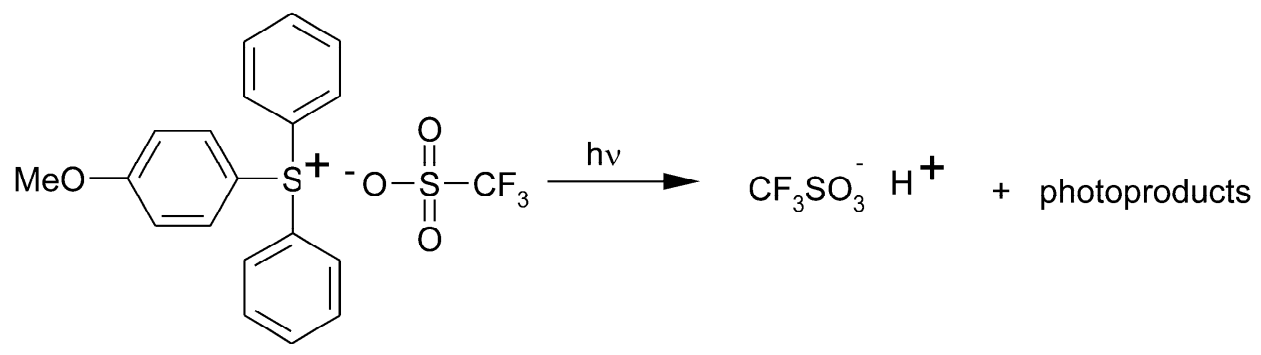

Scheme 2. Photochemical decomposition of (4-methoxyphenyl)-diphenylsulfonium triflate upon exposure to UV light inside a photoreactor leads to formation of triflic acid and other photoproducts.

In-situ generation of reagents may be useful for certain applications. The transparency of PDMS allows exposure to wavelengths down to $250 \mathrm{~nm}$, thus covering a wide interval. Should local generation of certain reagent be needed, exposure at precise points may be performed with laser beams, optical fibers or LED light sources.

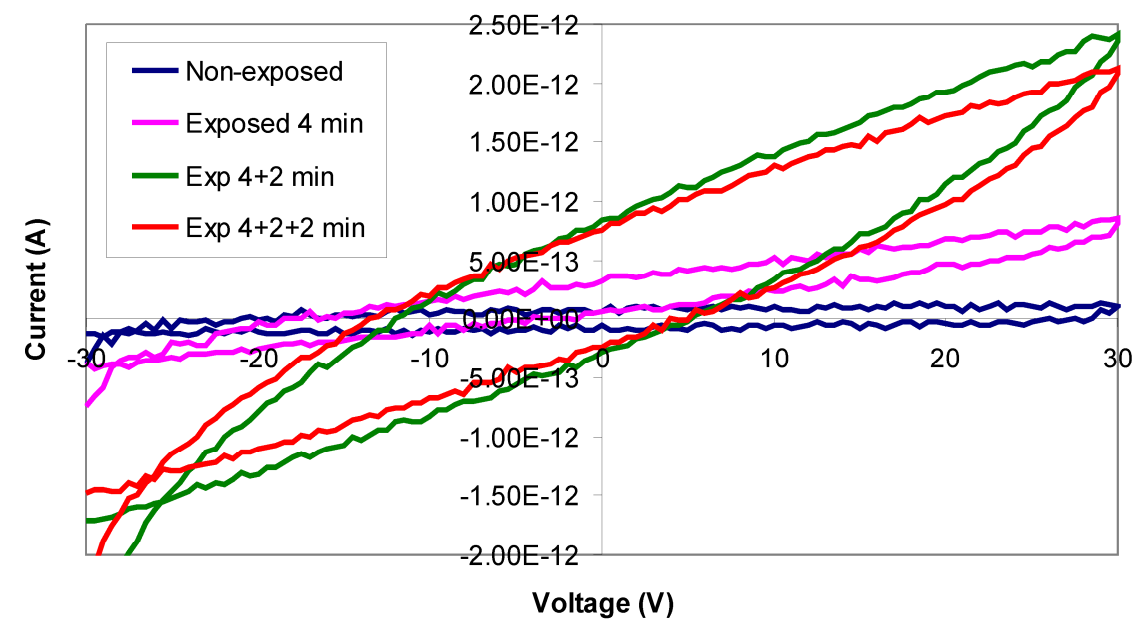

Fig.10. Current vs. voltage for a solution of (4-methoxyphenyl)-diphenylsulfonium triflate $1.58 \times 10^{-3} \mathrm{M}$ in ethanol, inside the microchannels, and exposed to UV radiation for $0,4,6$, and 8 minutes. 


\section{CONCLUSIONS}

Functional and versatile flexible PDMS microfluidics devices with potential application as micro-photoreactors and micro-electrolysis cells, and in detection of chemical and bio-relevant species were obtained. The PDMS flexible microfluidic device was successfully tested for optical and electrical detection of acid and ions. Owing to the transparency of PDMS and its chemical inertness, the device was successfully employed as a micro-photoreactor. The $\mathrm{Au}$ electrodes allow its use as a micro-electrolysis cell, opening the possibility for in-situ redox reactions at electrodes, with formation of novel reagents. For roll-to-roll manufacturing, polymers that cure faster than PDMS will have to be employed, and PMMA devices, already reported by others, [13] are a good candidate given the development of PMMAbased UV curable resist for imprint lithography. Current work in other groups at Holst Centre is focused on integration of in-house developed OLED light sources and light detectors in flexible optical sensors. Our next goal is to integrate the light source and the detector inside the microfluidic device, allowing a truly autonomous device, without the need of a spectrophotometer for spectroscopic measurements, and move towards fabrication in the roll-to-roll manner.

\section{ACKNOWLEDGEMENTS}

The research work was performed in the framework of the Holst Centre-TNO (The Netherlands). We gratefully acknowledge the contribution of the Holst Centre partners Philips, ASML, Singulus, and Polymer Vision to the research performed in the Lithography on Flexible Substrates group.

\section{REFERENCES}

[1] J.C. McDonald, G.M. Whitesides, "Poly(dimethylsiloxane) as a material for fabricating microfluidic devices", Acc. Chem. Res. 35/7 491-499 (2002)

[2] J. Pihl, J. Sinclair, M. Karlsson, O. Orwar, "Microfluidics for cell-based assays", Mat. Today 8/12 46-51 (2005)

[3] Z. Nie, W. Li, M. Seo, S. Xu, E. Kumacheva, "Janus and Ternary particles generated by microfluidic synthesis: design, synthesis, and self-assembly", J. Am. Chem. Soc. 128/29 9408-9412 (2006)

[4] G. Blanco-Gomez, A. Glidle, L.M. Flendrig, J.M. Cooper, "Integration of low-power microfluidic pumps with biosensors within a laboratory-on-a-chip device", Anal. Chem. 81 1365-1370 (2009)

[5] T.G. Henares, F. Mizutani, H. Hisamoto, "Current development in microfluidic immunosensing chip", Anal. Chim. Acta 611 17-30 (2008)

[6] K.-I. Ohno, K. Tachikawa, A. Manz, "Microfluidics: Applications for analytical purposes in chemistry and biochemistry", Electrophoresis 29 4443-4453 (2008)

[7] I.R. Lauks, "Microfabricated biosensors and microanalytical systems for blood analysis", Acc. Chem. Res. 31 317-324 (1998)

[8] Y. Hongbin, Z. Guangya, C.F. Siong, W. Shouhua, L. Feiwen, "Novel polydimethylsiloxane (PDMS) based microchannels fabrication method for lab-on-a-chip application", Sensors and Actuators B: Chem. 137 754-761 (2009)

[9] A.A.S. Bhagat, P. Hjothimuthu, I. Papautsky, "Photodefinable polydimethylsiloxane (PDMS) for rapid lab-ona-chip prototyping", Lab Chip 7 1192-1197 (2007)

[10] K. Tsougeni, A. Tserepi, E. Gogolides, "Photosensitive poly(dimethylsiloxane) materials for microfluidic applications", Microel. Eng. 84 1104-1108 (2007)

[11] X. Liu, Q. Wang, J. Qin, B. Lin, "A facile "liquid-molding" method to fabricate PDMS microdevices with 3dimensional channel topography", Lab Chip/9 1200-1205 (2009)

[12] H. Becker, C. Gartner, "Polymer microfabrication technologies for microfluidic systems", Anal. Bioanal. Chem. 390 89-111 (2008)

[13] Y. Chen, L. Zhang, G. Chen, "Fabrication, modification, and application of poly(methyl methacrylate) microfluidic chips", Electrophoresis 29 1801-1814 (2008)

[14] D.K. Maurya, W.Y. Ng, K.A. Mahabadi, Y.N. Liang, I. Rodriguez, "Fabrication of lab-on chip platforms by hot embossing and photo patterning", Biotechnol. J. 2 1381-1388 (2007)

[15] M. Peter, T. Schuler, F. Furthner, P. Rensing, G.T. van Heck, H.F.M. Schoo, R. Moller, W. Fritzsche, A.J.J.M. van Breemen, E.R. Meinders, "Flexible Biochips for Detection of Biomolecules", Langmuir 25/9 5384-5390 (2009)

[16] Y. Berdichevsky, J. Khandurina, A. Guttman, Y.-H. Lo, "UV/ozone modification of poly(dimethylsiloxane) microfluidic channels", Sensors and Actuators B: Chem. 97 402-408 (2004) 
[17] M. Peter, F. Furthner, J. Deen, W.J.M. de Laat, E.R. Meinders, "Lithographic patterning of metals on flexible plastic foils", Thin Solid Films 517 3081-3086 (2009)

[18] H. Hillborg, J.F. Ankner, U.W. Gedde, G.D. Smith, H.K. Yasuda, K. Wilkstrom, "Crosslinked polydimethylsiloxane exposed to oxygen plasma studied by neutron reflectometry and other surface specific techniques", Polymer 41 6851-6863 (2000)

[19] J.C. Scaiano, M. Laferriere, M.G. Ivan, G.N. Taylor, "A Protocol for the Verification of Acid Generation in 157 nm Lithography", Macromolecules 366692 (2003)

[20] S. Corrent, P. Hahn, G. Pohlers, T.J. Connoly, J.C. Scaiano, V. Fornes, H. Garcia, "Intrazeolite photochemistry. 22 Acid-base properties of Coumarin 6. Characterization in solution, the solid state, and incorporated into supramolecular systems", J. Phys. Chem. B 102 5852-5858 (1998) 